\title{
Numerous GABAergic Afferents to Locus Ceruleus in the Pericerulear Dendritic Zone: Possible Interneuronal Pool
}

\author{
Gary Aston-Jones, ${ }^{1}$ Yan Zhu, ${ }^{1}$ and J. Patrick Card ${ }^{2}$ \\ ${ }^{1}$ Department of Psychiatry, University of Pennsylvania, Philadelphia, Pennsylvania 19104, and ${ }^{2}$ Department of Neuroscience, University of Pittsburgh, \\ Pittsburgh, Pennsylvania 15260
}

\begin{abstract}
Most nuclei in the CNS are composed of principal neurons that project to other areas and interneurons that serve to integrate information among afferents. The noradrenergic brain nucleus locus ceruleus (LC) has appeared to be an exception to this general rule, because the LC is composed almost entirely of noradrenergic principal neurons. Here, we report that numerous small neurons in the peri-LC region become retrogradely labeled after focal injections of wheat germ agglutinin-apo (inactivated) horseradish peroxidase conjugated to colloidal gold, or pseudorabies virus (PRV), into the nuclear core of the rat LC. A substantial number of these neurons were routinely found within the dendritic field of the LC, in the area surrounding the compact cell-dense region classically defined as LC. Double labeling revealed that a large percentage of these cells stained for GABA. Ultrastructural analyses revealed axodendritic and axosomatic contacts between PRV-labeled afferents and LC neurons labeled with tyrosine hydroxylase immunohistochemistry. In addition, PRV-labeled neurons or axons were immunopositive for GABA in ultrastructural localizations. Analysis of the synaptology of immunopositive profiles demonstrated that these LC afferents in the peri-LC region receive several non-LC synaptic inputs. These results indicate that a population of small GABAergic neurons in the peri-LC dendritic zone may provide interneuronal integration for LC noradrenergic neurons.
\end{abstract}

Key words: norepinephrine; locus ceruleus; GABA; ultrastructure; interneurons; pseudorabies virus

\section{Introduction}

The brain nucleus locus ceruleus (LC) is the largest source of norepinephrine (NE) in the CNS. The highly divergent efferents of the LC innervate all levels of the neuraxis and provide the sole source of NE to cerebral and cerebellar cortices and hippocampus. This broad projection system implies that the LC has a global function in behavior, and this system has been implicated in several such functions, including sleep/waking regulation, learning, memory and cognitive performance (for review, see Aston-Jones et al., 2000; Berridge and Waterhouse, 2003).

Knowledge of origins of inputs to LC neurons is important for better understanding the functions of this noradrenergic system. Several studies of afferents have identified direct inputs from sources including the rostral medulla and hypothalamus, as well as indirect inputs from the suprachiasmatic nucleus (Cedarbaum and Aghajanian, 1978; Pollard et al., 1978; Aston-Jones et al., 1986, 2001; Ennis and Aston-Jones, 1988, 1989; Pieribone et al., 1988; Horvath et al., 1999; Steininger et al., 2001). However, several CNS areas, including the periaqueductal gray, medial prefrontal cortex, and nucleus tractus solitarius, send projections into the region immediately surrounding the LC nucleus (AstonJones et al., 1995). We have termed this region the pericerulear

Received Dec. 3, 2003; revised Jan. 10, 2004; accepted Jan. 14, 2004

This work was supported by United States Public Health Service Grant NS 24698. We thank Drs. R. Miselis and L. Enquist for generously supplying PRV and PRV antibodies.

Correspondence should be addressed to Dr. Gary Aston-Jones, Department of Psychiatry, University of Pennsylvania, 705 Stellar-Chance/6100, 422 Curie Boulevard, Philadelphia, PA 19104. E-mail: gaj@mail.med.upenn.edu. DOI:10.1523/JNEUROSCI.5339-03.2004

Copyright $\odot 2004$ Society for Neuroscience $\quad$ 0270-6474/04/242313-09\$15.00/0 dendritic zone because it contains a dense plexus of LC dendrites (Swanson, 1976; Shipley et al., 1996). The LC dendrites that define this region are extensive, and it therefore represents an important site through which afferents may modulate the activity of LC neurons. Localization of GABAergic neurons within the peri-LC dendritic field (Ijima and Ohtomo, 1988; Jones, 1991) also raises the possibility that interneurons in this region contribute to the functional modulation of LC activity. This possibility is supported by the demonstration that the LC is densely innervated by GABA fibers (Berod et al., 1984; Mugnaini and Oertel, 1985; Ennis and Aston-Jones, 1989; Aston-Jones et al., 1991). Collectively, these observations suggest that GABA input to LC neurons from these local neurons in the peri-LC zone might constitute a previously unrecognized interneuronal pool for the LC. This would represent a potentially significant element in the integration of afferent inputs that regulate activity in the LC-NE system.

To examine this possibility, here we examined retrograde labeling in the peri-LC zone after focal microinjections of retrograde tracers into the LC nuclear core. We also examined GABAergic neurons and LC dendrites in the peri-LC zone at the ultrastructural level to determine possible direct synaptic relationships. Our results indicate that the GABA neurons in the peri-LC zone provide a substantial input to LC extranuclear dendrites, and may represent a set of interneurons serving to integrate inputs to the LC system.

\section{Materials and Methods}

Animal surgery and microinjections

Thirty-four adult male Sprague Dawley rats (300-400 gm; specific pathogen-free; Charles River Laboratories, Wilmington, MA) were used. 
They were anesthetized with Nembutal (50 mg/ $\mathrm{kg}$, i.p.) and placed in a stereotaxic apparatus. All surgical procedures conformed to the BioSafety level 2 regulations for studies involving the use of infectious pathogens [United States Department of Health and Human Services Publication No. (Centers for Disease Control and Prevention) 88-8395; Biosafety in Microbiological and Biomedical Laboratories)] and were approved by the University of Pennsylvania Institutional Animal Care and Use Committee. The LC was localized for microinjections with electrophysiological guidance as described previously (Aston-Jones et al., 1986). The retrograde tracer wheat germ agglutinin conjugated apo (inactivated) horseradish peroxidase coupled with colloidal gold (WGA-Au; 300-600 $\mathrm{nl}$ ), or the retrograde trans-synaptic tracer pseudorabies virus (PRV, Bartha strain; $60 \mathrm{nl}$, $607 \mathrm{pfu} / \mathrm{nl})$, was microinjected unilaterally into one LC through a glass pipette ( $20 \mu \mathrm{m}$ tip) over $10 \mathrm{~min}$ by brief pneumatic pulses (Picrospritzer; General Valve, Fairfield, NJ) (Drolet et al., 1992; Chen et al., 1999; AstonJones and Card, 2000). Cholera toxin subunit B (CTb, 0.05\%; Sigma, St. Louis, MO) was added to the PRV solution in some cases, but did not interfere with PRV tracing (Chen et al., 1999; Aston-Jones and Card, 2000). Previous analysis has demonstrated that the injection of WGA-Au or PRV in this manner can produce injection sites that are restricted mainly to the LC nuclear core, with little diffusion into the peri-LC zone. In addition, previous ultrastruc-

tural analysis has demonstrated that progeny virus fills the entire somatodendritic compartment of infected neurons, including the most distal dendrites (Card et al., 1993; Carr et al., 1999). Thus, these injections would lead to retrograde transneuronal spread of virus through afferents contacting the extranuclear dendrites of infected LC neurons. This retrograde trans-synaptic transfer of PRV from dendrites to afferents provides a powerful and unique way to identify inputs specifically to distal LC dendrites located in the peri-LC zone.

\section{Perfusion and tissue processing}

Light microscopy. After 4-5 d (WGA-Au injections) or 23-39 hr (PRV injections) of survival time, animals were deeply anesthetized with Nembutal and transcardially perfused with saline followed by $4 \%$ paraformaldehyde. Brains were removed and placed in the same fixative for $2 \mathrm{hr}$, then transferred to $20 \%$ sucrose overnight. Coronal sections (20-40 $\mu \mathrm{m}$ thick) were cut on a cryostat throughout the rostrocaudal extent of the rostral brainstem and collected in $0.1 \mathrm{M}$ PBS.

Methods for light-level immunohistochemistry are similar to those published previously from our laboratory and others, and are described here only briefly.

WGA-Au histochemistry. Sections from 10 rats were incubated in Intense BL silver enhancement solution (Amersham Biosciences, Arlington Heights, IL) for $30 \mathrm{~min}$ at room temperature to visualize WGA-Au, as described previously (Drolet et al., 1992).

Tyrosine hydroxylase immunohistochemistry. Tissue was incubated in a monoclonal mouse anti-tyrosine hydroxylase $(\mathrm{TH})$ antibody (1:6000; Incstar, Stillwater, MN) in PBS plus $0.3 \%$ Triton X-100 (PBST) overnight, at room temperature. The specificity of this antibody has been established in previous studies (Pickel et al., 1975). For bright-field staining, after incubation in the primary antibody sections were placed in a donkey anti-mouse IgG (1:500; The Jackson Laboratory, Bar Harbor, ME) in PBST for 90 min at room temperature, and incubated with mouse peroxidase-antiperoxidase (1:500 in PBST; The Jackson Laboratory) for $90 \mathrm{~min}$ at room temperature. Incubation of the tissue in Tris buffer containing diaminobenzidine and $\mathrm{H}_{2} \mathrm{O}_{2}$ for 5-10 min produced the im- munoperoxidase reaction product. For fluorescence staining, after primary incubation (1:1000) sections were placed in donkey anti-mouse IgG conjugated with Alexa Fluor 488 in PBST for $2 \mathrm{hr}$ at room temperature (1:200; Molecular Probes, Eugene, Or).

Glutamic acid decarboxylase immunohistochemistry. Immunohistochemistry for glutamic acid decarboxylase (GAD), the enzyme responsible for GABA synthesis, was used in light microscopic studies to tentatively identify GABAergic neurons. Tissue was incubated overnight in a sheep anti-GAD antibody (1:2000; gift from M. L. Tappaz, Lyon, France) in PBS (without Triton X-100 to reveal fibers) at room temperature. Specificity of the antiserum for GAD has been established in previous studies (Oertel et al., 1982; Berod et al., 1984). For bright-field staining, after incubation in the primary antibody sections were incubated in a biotinylated donkey anti-sheep IgG (1:500; The Jackson Laboratory) in PBS for $90 \mathrm{~min}$ at room temperature followed by $\mathrm{ABC}$ Elite reagents (1:1000; Vector Laboratories, Burlingame, CA) diluted in PBS for 90 min at room temperature. The immunoperoxidase reaction product was revealed by incubation of sections in a DAB plus $\mathrm{H}_{2} \mathrm{O}_{2}$ mixture in Trisbuffered saline (TBS) for 5-10 min. For fluorescence staining, after primary incubation $(1: 1000)$ sections were placed in a donkey anti-sheep IgG conjugated with Alexa Fluor 594 in PBS for $2 \mathrm{hr}$ at room temperature (1:200; Molecular Probes).

PRV immunohistochemistry. Tissue from five rats was incubated in a polyclonal goat anti-PRV antibody (GB-320; gift from R. Miselis, Philadelphia, PA) 1:20,000 in PBST overnight at room temperature. After incubation in primary antiserum, sections were incubated in biotinylated donkey anti-goat IgG (1:1000 in PBST; The Jackson Laboratory) for 90 min at room temperature followed by $\mathrm{ABC}$ Elite reagents (1:1000 in PBST; Vector Laboratories) for $90 \mathrm{~min}$ at room temperature. Visualization of the immunoperoxidase signal was accomplished by incubation in the blue substrate SG (Vector Laboratories) for 5-10 min.

Electron microscopy. After 23-39 hr of survival time following PRV injections, animals were deeply anesthetized with Nembutal and transcardially perfused with $50 \mathrm{ml}$ saline and heparin $(1000 \mathrm{U} / \mathrm{ml})$ followed by $50 \mathrm{ml}$ of $3.8 \%$ acrolein and $300 \mathrm{ml}$ of $2 \%$ paraformaldehyde for $3-5$ 


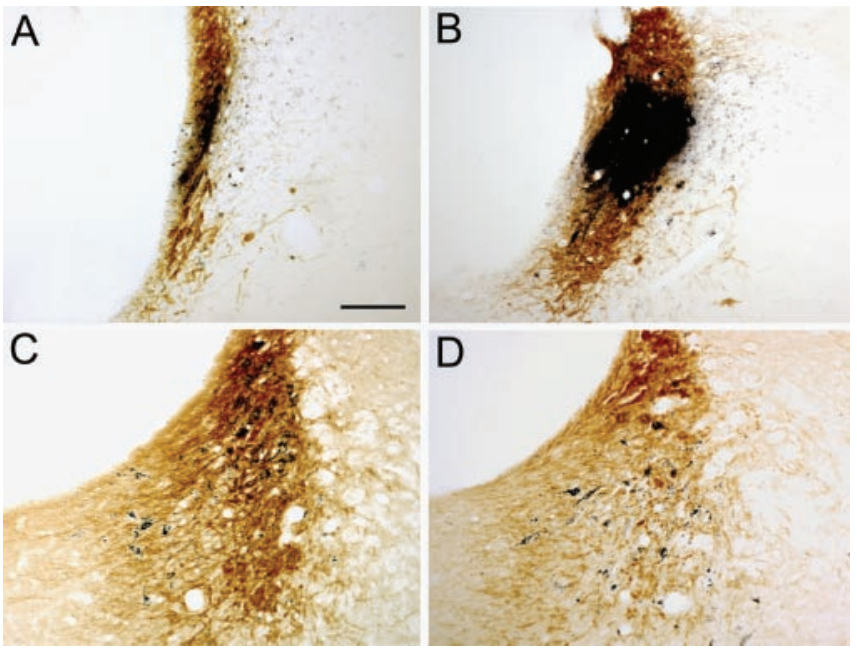

Figure 2. Injection of WGA-Au into $\mathrm{LC}$ reveals local afferents. Photomicrographs of frontal sections through the $\mathrm{LC}$ showing a WGA-Au injection ( $A$, B, black) confined to within the mid-LC and caudal $\mathrm{LC}$ nucleus. NE neurons in $\mathrm{LC}$ are stained with an antibody against TH (brown). Note the focal WGA-Au injection restricted to the LC nucleus. Note also the retrogradely labeled darkly stained neurons among the $\mathrm{LC}$ dendrites, most apparent among the $\mathrm{LC}$ extranuclear dendrites in C and D. The distribution of these neurons is primarily coextensive with that of the $\mathrm{LC}$ dendrites. Scale bar, $200 \mu \mathrm{m}$.

$\min$. The brains were removed and placed in the same fixative for $2 \mathrm{hr}$, then transferred to $0.1 \mathrm{M}$ phosphate buffer (PB). Quasi-horizontal sections (40 $\mu \mathrm{m}$ thick) were cut with a Vibratome, washed in $\mathrm{PB}$, and incubated in $1 \%$ sodium borohydride in $\mathrm{PB}$ for $30 \mathrm{~min}$ to remove reactive aldehydes. Sections were then incubated in $5 \%$ bovine serum albumin (BSA) in $0.1 \mathrm{~m}$ TBS for another $30 \mathrm{~min}$.

Methods for dual pre-embedding immunocytochemistry were as described previously (Chan et al., 1990) and are described here only briefly.

PRV plus GABA dual immunocytochemistry. Tissue from 13 rats was incubated in a cocktail of mouse anti-GABA (1:1000; Sigma) and rabbit anti-PRV antibodies (1:4000; gift from L. Enquist, Princeton University, Princeton, NJ) overnight, at room temperature. Specificity of these reagents has been established previously (Card et al., 1990). After incubation in the cocktail, sections were reacted in biotinylated donkey antirabbit IgG (1:400 in TBS plus 0.1\% BSA; The Jackson Laboratory) for 30 min, followed by ABC Elite reagents (1:400 in TBS; Vector Laboratories) for $30 \mathrm{~min}$, both at room temperature. Tissue was then reacted in a DAB plus $\mathrm{H}_{2} \mathrm{O}_{2}$ TBS solution for 4-6 min. Next, sections were incubated in $0.01 \mathrm{M}$ PBS plus $0.8 \%$ BSA plus $0.1 \%$ gelatin for $30 \mathrm{~min}$, followed by goat anti-mouse IgG conjugated to $1 \mathrm{~nm}$ gold particles (1:50; Amersham) for $2 \mathrm{hr}$ at room temperature. Sections then were placed in $2 \%$ glutaraldehyde in $0.01 \%$ PBS for $10 \mathrm{~min}$, and subsequently rinsed in $0.2 \mathrm{M}$ sodium citrate buffer. Finally, silver intensification (Amersham silver enhancement kit) was carried out for 8-10 min.

PRV plus TH dual immunocytochemistry. Procedures were the same as above for PRV plus GABA dual immunocytochemistry, except for the use of a monoclonal mouse anti-TH antibody (1:1000; Incstar; $n=13$ rats).

GABA plus TH dual immunocytochemistry. Procedures were the same as for PRV plus TH dual immunocytochemistry, except for the use of a monoclonal mouse anti-GABA antibody (1:1000; Sigma; $n=6$ rats).

After staining, all electron microscopic (EM) sections were postfixed for $1 \mathrm{hr}$ in $2 \%$ osmium tetroxide in $\mathrm{PB}$, dehydrated through a graded series of alcohols and propylene oxide, and flat-embedded in Epon 812 (Electron Microscopy Science, Fort Washington, PA).

Localization of field of analysis. Considerable effort was made to correlate light and electron microscopic observations to ensure that analysis was restricted to the peri-LC dendritic field. The precise location of the field of analysis is described in the following section. The organization of the analysis and the method of sampling, illustrated in Figure 6, were conducted in the following manner. All flat-embedded Vibratome sec-
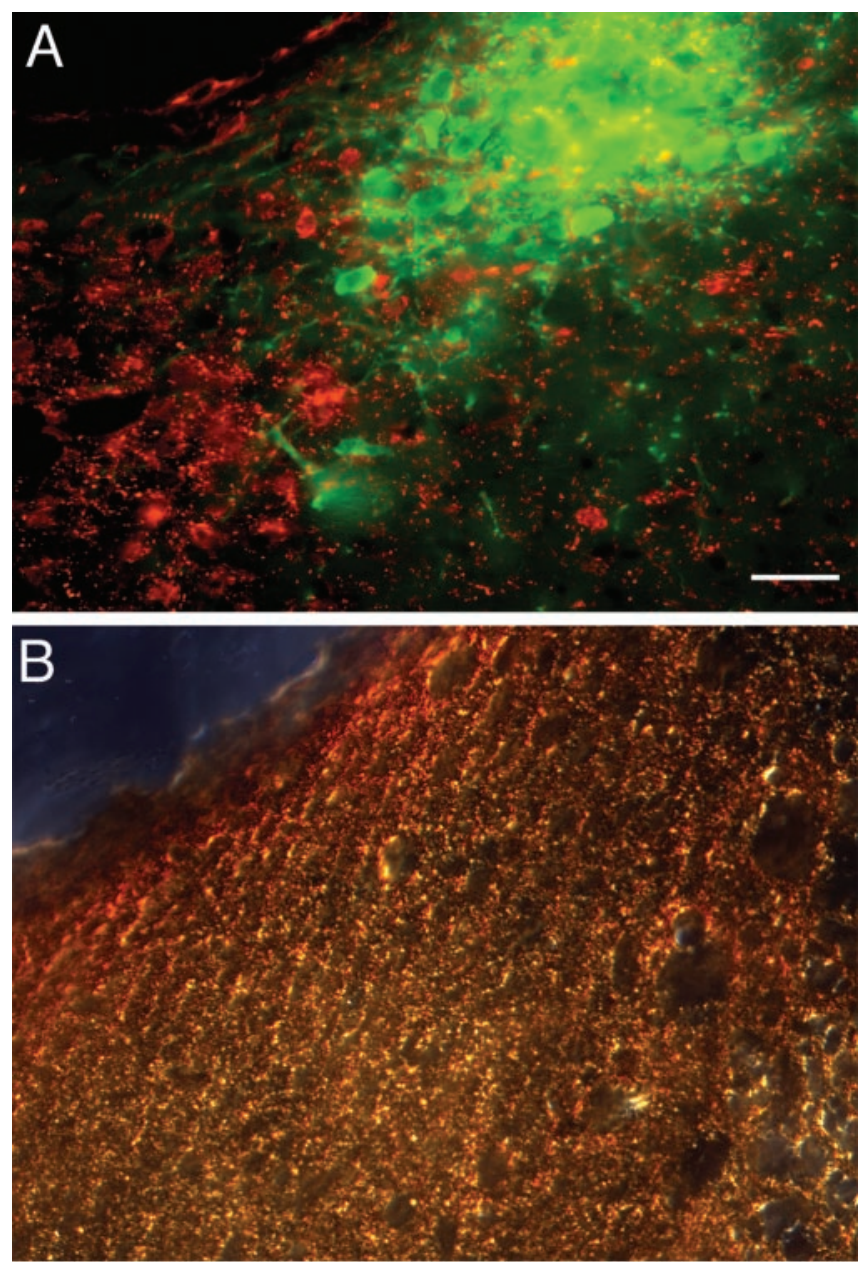

Figure 3. GABAergic neurons and terminals in peri-LC. $A$, Fluorescence photomicrograph of a frontal section through the level of the mid-LC showing GAD + (presumably GABAergic; Alexa Fluor 594; red) and TH + (Alexa Fluor 488; green) neurons and processes in the peri-LC and LC nuclear core. This section was stained to emphasize the GAD + staining of somata (no Triton $\mathrm{X}-100$ pretreatment). Note the numerous $\mathrm{GAD}+$ neurons adjacent to the ventromedial NE neurons and the dense GAD + terminals in both peri- $L C$ and the $L C$ nucleus (more clearly revealed in $B$ ). Medial is to the left, dorsal is up. The fourth ventricle is at the top left. $B$, Dark-field photomicrograph through the same area as in $A$ from a Vibratome section processed to emphasize GAD + fibers and terminals ( $0.3 \%$ Triton X-100 pretreatment). Note the dense GAD+ innervation in the peri- $\mathrm{LC}$ and the $\mathrm{LC}$ nuclear core. $\mathrm{LC}$ neurons are visible as dark unstained somata in the upper right. The orientation for both panels is identical. Scale bar, $50 \mu \mathrm{m}$.

tions were transilluminated and examined with a stereomicroscope to localize reaction product. Sections that contained the desired portion of the peri-LC dendritic field were photographed. The immunolabeled peri-LC tissue was then cut from the Vibratome section, glued to a blank resin stub, and trimmed to the area of interest using stereo-optics of a Leica (Deerfield, IL) Ultracut E ultramicrotome. Sequential series of semi-thin $(0.35 \mu \mathrm{m})$ and ultrathin $(70-80 \mathrm{~nm})$ sections were collected on microscope slides and Formvar-coated slot grids, respectively. Semithin sections were stained with toluidine blue, and ultrathin sections were stained with heavy metals as described previously (Card et al., 1993). Sections were collected such that each series of ultrathin sections (generally 5-20 sections) was bracketed by semi-thin sections. In every case, the closest semi-thin section was photographed and used as a map to direct the ultrastructural analysis. Landmarks (e.g., blood vessels and the ependymal lining of the fourth ventricle) permitted precise localization of the region of interest. Individual immunoreactive profiles apparent in the semi-thin sections could then be located in the ultrathin sections.

A systematic sampling strategy was used for each ultrathin section. 
After identification of landmarks identifiable in the semi-thin section, the peri-LC region was completely sampled [using a Zeiss (Oberkochen, Germany) 10A transmission electron microscope] with sequential passes using the sampling strategy detailed by Rinaman et al. (1989). Representative samples of all immunolabeled profiles and their synaptic relations were photographed, and their location was recorded on the maps derived from the semi-thin sections. Photographic enlargements $(8 \times 10$ inches $)$ of each field were printed and analyzed to produce the data that forms the basis of this report.

\section{Results}

\section{Light microscopic observations}

As reported previously (Swanson, 1976; Shipley et al., 1996), dendrites of LC neurons extended far into the pericerulear parenchyma. Low-power images of sections through the LC and adjacent areas stained for $\mathrm{TH}$ revealed $\mathrm{NE}$ processes that extended from the cell-dense region of LC for hundreds of microns into the peri-LC area (Fig. 1). Our previous report demonstrated that the largest accumulations of extranuclear LC dendrites are found rostroventromedial and caudodorsolateral to the LC nuclear core (Shipley et al., 1996). This study also revealed that $>90 \%$ of these processes in the rostroventromedial peri-LC zone are dendrites. Examination of this region revealed that it contains numerous GABAergic neurons (described below) and we therefore selected it as the focus of the present analysis. As in previous studies, we found that dendrites of LC neurons in this peri-LC zone were best revealed in quasi-horizontal sections cut dorsocaudally to ventrorostrally, which follows the axis of most of these processes.

\section{Retrogradely labeled LC afferents in the peri-LC zone: WGA-Au labeling}

Microinjections of WGA-Au into the LC in 10 cases produced focal deposits that were limited to the LC nuclear core and did not extend into the peri-LC zone (Fig. 2). This restricted spread of WGA-Au was characteristic of all survival times, because similar injection sites were found for the same volume injections after 5 hr, $48 \mathrm{hr}, 3 \mathrm{~d}$, and $5 \mathrm{~d}$ (Y. Zhu and G. Aston-Jones, unpublished observations).

Focal WGA-Au deposits in the LC nuclear core produced numerous retrogradely labeled neurons in the peri-LC dendritic zone in all cases. As shown in Figure 2, labeled neurons were preferentially located among $\mathrm{TH}$-immunopositive LC dendrites in the rostroventral peri-LC zone. These labeled cells typically had small $(\sim 8 \times 15 \mu \mathrm{m})$ round or oval perikarya.

\section{Dual labeling for WGA-Au and GABA}

Figure 3 shows sections through the peri-LC stained with an antibody against GAD to reveal GABAergic neurons and processes. These photos reveal a dense GABA fiber innervation of the ventromedial peri-LC zone, which is defined in Figure $3 A$ by $\mathrm{TH}$ immunofluorescence. Analyses of bright-field illuminated sections of similar regions from animals containing focal injections of WGA-Au in the LC nuclear core revealed that retrogradely labeled (WGA-Au+) cells were also frequently GAD + (Fig. 4A$D)$. Counts of neurons in 12 sections from three rats demonstrated that $\sim 45 \%$ of WGA-Au-labeled afferent somata to the LC in the peri-LC zone also stained for GAD.

\section{Retrogradely labeled LC afferents in the peri-LC zone after LC PRV injection}

Microinjection of PRV into the LC nuclear core in 18 cases produced numerous retrogradely labeled neurons in the peri-LC zone, as illustrated in Figure 5. Note that at both $23 \mathrm{hr}$ (Fig. $5 A-D$ ) and $35 \mathrm{hr}$ (Fig. $5 A^{\prime}-D^{\prime}$ ) of survival, PRV-labeled neurons in the peri-LC were primarily located in the peri-LC dendritic zone, as found for WGA-Au labeling in Figures 2 and 4. Quantitative analysis of PRV labeling after LC injections revealed that neurons were much more numerous at $35 \mathrm{hr}$ of survival than at $23 \mathrm{hr}(188.2 \pm 20.5$ vs $74.8 \pm 9.7$ cells per section, respectively; $n=6$ sections in each of two animals at each survival time). These results indicate that peri-LC labeling is mediated by PRV transport and replication rather than by the spread of injection outside of the LC. This is consistent with previous studies showing that PRV is avidly taken up at the site of injection and that the affinity of viral envelope proteins for extracellular matrix molecules further restricts the spread of virus from the site of injection (Card et al., 1999).

PRV injections also produced retrograde infection of neurons with direct inputs to the LC, such as the lateral paragigantocellularis (PGi), as identified previously with conventional monosynaptic retrograde tracers (Aston-Jones et al., 1986; Van Bockstaele et al., 1998). Here, we found that the amount of retrograde labeling in the PGi at $23 \mathrm{hr}$ after a PRV injection in the LC is similar to 

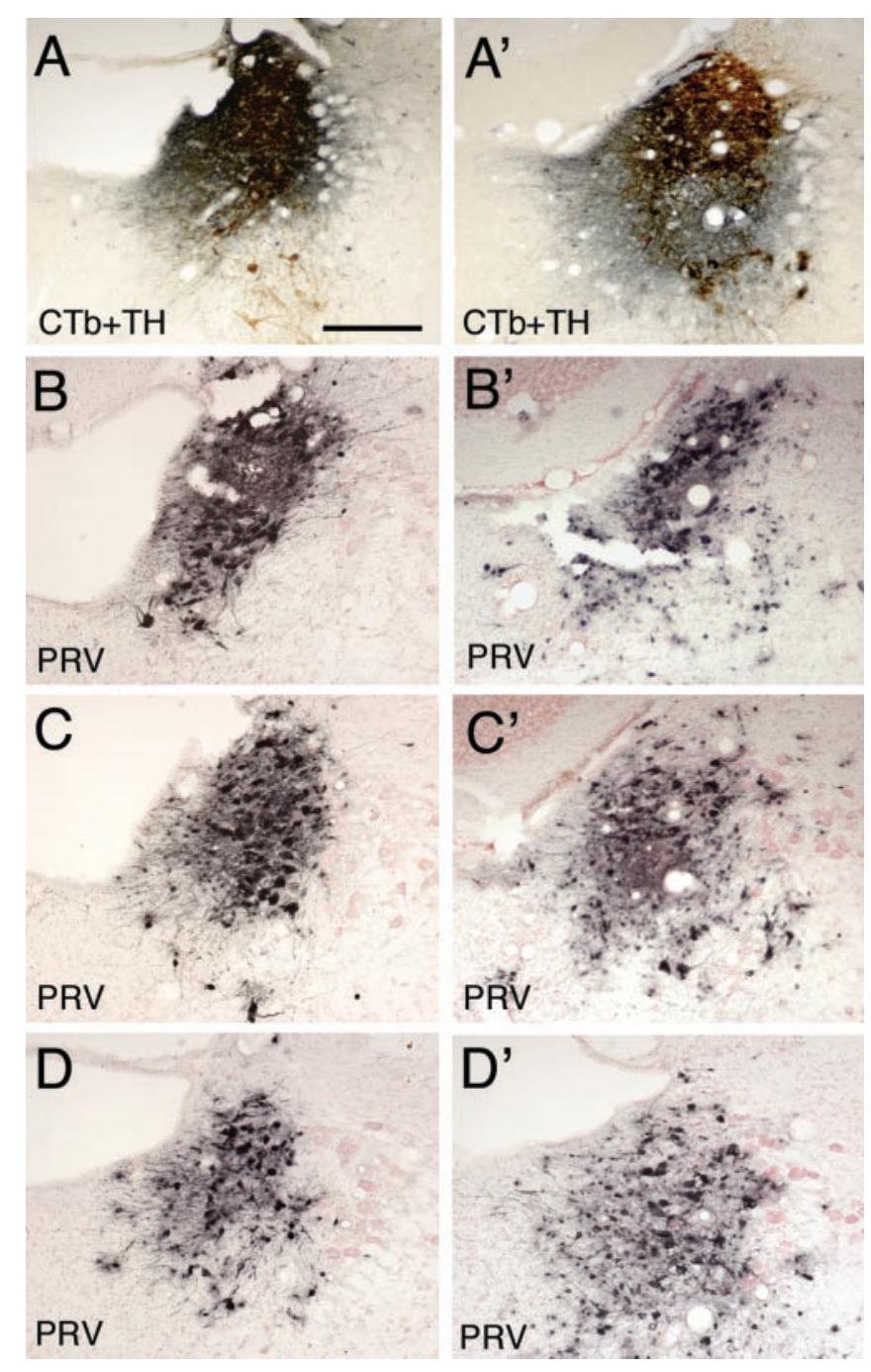

Figure 5. Peri-LC labeling with PRV. Photomicrographs of frontal sections through the $\mathrm{LC}$ at $23 \mathrm{hr}(A-D)$ or $35 \mathrm{hr}\left(A^{\prime}-D^{\prime}\right)$ after focal microinjections of the trans-synaptic retrograde tracer PRV in the caudal LC nuclear core. $A, A^{\prime}$, Injection sites in LC indicated by co-injected CTb. CTb was used for this because PRV is quickly transported from the site of injection, so that the injection site is best delineated with a co-injected agent. O'Donnell et al. (1997) demonstrated that CTb is taken up from a larger area than PRV after simultaneous injection, so the zone of PRV uptake would be smaller than that evident for CTb. Note the dense injection focused in the LC nuclear core in both cases. $B-D, B^{\prime}-D^{\prime}$, Sections through the caudal $\left(B\right.$ and $\left.B^{\prime}\right)$, mid- ( $C$ and $C^{\prime}$ ) and rostral $\left(D\right.$ and $\left.D^{\prime}\right) \mathrm{LC}$ at 23 or $35 \mathrm{hr}$ of survival $\left(B-D\right.$ and $B^{\prime}-D^{\prime}$, respectively) after the injections indicated in $A$ and $A^{\prime}$. Note the greater number of small, labeled neurons in the peri-LC zone at 35 compared with $23 \mathrm{hr}$, particularly at rostral levels ( $D$ and $\left.D^{\prime}\right)$. These findings indicate that there are two paths for labeling in the peri-LC: a nuclear path mediated by projections of peri-LC neurons into the LC nuclear core and a dendritic path mediated by peri-LC neuronal connections onto extranuclear LC dendrites. For all panels, ventral is down, medial is left; the fourth ventricle is at the top left. Scale bar, $200 \mu \mathrm{m}$.

that found after a WGA-Au injection in the LC (Drolet et al., 1992; Aston-Jones et al., 2001). Thus, we interpret the differing magnitude of infection of peri-LC neurons at 23 and $35 \mathrm{hr}$ as evidence for two populations of neurons with synaptic relations with the LC. Specifically, we postulate that the neurons observed at $23 \mathrm{hr}$ were infected by virtue of their projections into the nuclear core, and the larger numbers that became apparent at $35 \mathrm{hr}$ were infected through their synaptic connections with LC dendrites in the peri-LC dendritic zone. This time course is consistent with the replication cycle of PRV (Enquist et al., 1999).
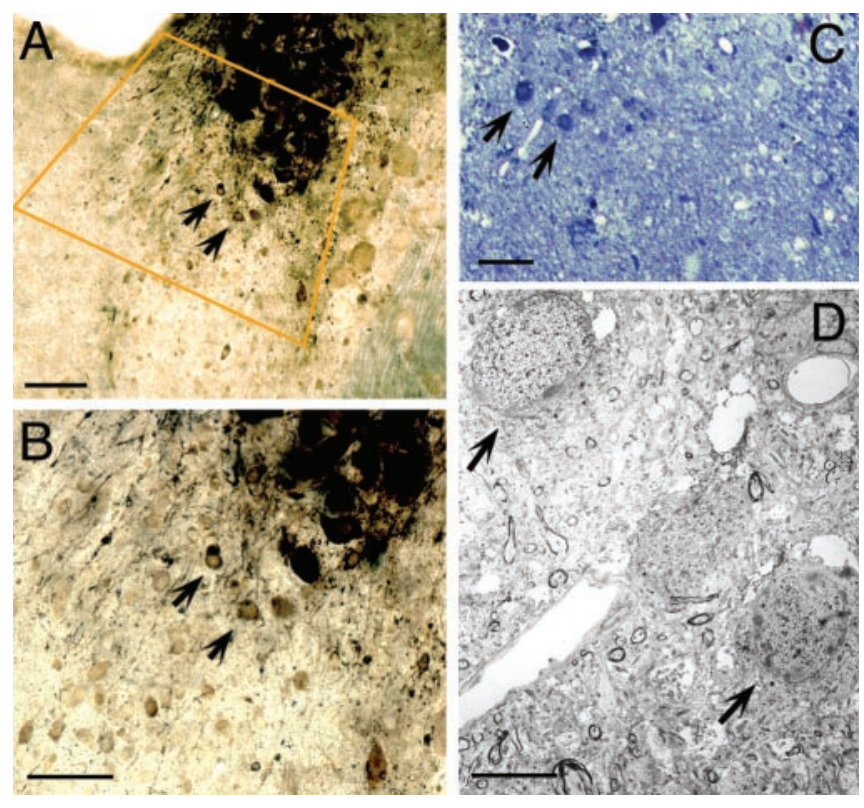

Figure 6. Method used to identify peri-LC region for ultrastructural analyses. Systematic analysis of sections from Vibratome and ultramicrotome allowed unambiguous identification of peri-LC region in EM analyses. $A, B$, Photomicrographs of a transilluminated quasi-horizontal 40 $\mu \mathrm{m}$ thick Vibratome section through the $L C$ at $35 \mathrm{hr}$ survival after a focal microinjection of PRV in the LC nucleus. This section is doubly stained for PRV (DAB, brown) and TH (silver-intensified gold, black). The trapezoid defined in $A$ illustrates the rostroventral peri-LC region sectioned for subsequent ultrastructural analyses, shown at higher magnification in $B$. Note the numerous small PRV-labeled neurons among TH dendrites in the peri-LC. Rostroventral is down, medial is left; the fourth ventricle is at the top left. Arrows indicate the same cells in both $A$ and $B$. $C$, Toluidine blue-counterstained $0.35 \mu \mathrm{m}$ semi-thin section from the trapezoid identified in $A$. Arrows indicate the same cells as in $A$ and $B$. D, Ultrathin $(70 \mathrm{~nm})$ section adjacent to the semi-thin section shown in C. Scale bars: $A, 100 \mu \mathrm{m} ; B, C, 50 \mu \mathrm{m} ; D, 10 \mu \mathrm{m}$.

\section{Ultrastructural observations}

The method used to identify and systematically examine the peri-LC dendritic field in ultrastructural analysis is described in Materials and Methods and illustrated in Figure 6. Using this approach, we were able to follow specific regions of the peri-LC area from the low-power light microscopic level to high-power electron microscopic images. This ensured that our ultrastructural analysis was focused specifically on the peri-LC dendritic area.

As shown in Figure 7, we obtained frequent images of GABA+ neuronal elements in the peri-LC region that contained PRV particles after PRV injections into the LC nuclear core. In counts of EM material, we found that 101 of 131 (77\%) PRV-labeled neurons in the peri-LC stained for GABA (counts obtained from 10 thin sections cut from four blocks in two rats). The fine structural features of these viral profiles conformed to those established in previous studies of PRV replication (Whealy et al., 1991; Card et al., 1993). For example, the nuclei of the cells contained viral capsids and the cytoplasm and contiguous dendrites frequently contained enveloped virions. It is important to note that there was no evidence of cytopathic changes in infected neurons in any case, irrespective of post-inoculation survival.

GABA terminals frequently made symmetrical synaptic contacts onto $\mathrm{PRV}+$ or $\mathrm{TH}+$ dendrites in the peri-LC region (Fig. 8). Some of these GABA terminals contained PRV particles, as did some of the $\mathrm{TH}+$ dendrites that received input from GABA terminals. These results are consistent with the interpretation that PRV virions were transported into peri-LC GABA neurons from 
LC dendrites, indicating a synaptic connection from local peri-LC GABA neurons to LC noradrenergic neurons.

GABA + dendrites in the peri-LC received numerous symmetrical and asymmetrical synaptic inputs, as illustrated in Figure 9. Contacts were analyzed on 226 peri-LC GABA dendrites from eight blocks in six rats. Results revealed that of 293 contacts from non-GABA (unlabeled) terminals onto GABA dendrites in this region, $25(8.5 \%)$ were symmetric, and 50 (17\%) were asymmetric; the remainder were nonsynaptic appositions. In addition, numerous GABA inputs to GABA dendrites were also observed (Fig. 9). Of 155 GABA terminals onto GABA dendrites, 29 (19\%) made symmetrical synapses and $12(8 \%)$ were asymmetric; the remainders were nonsynaptic appositions.

Dendrites that did not stain for either GABA or $\mathrm{TH}$ were also frequently observed in this peri-LC region. Analyses indicated that $\sim 25 \%$ of dendritic profiles in the peri-LC were neither $\mathrm{GABA}+$ nor $\mathrm{TH}+$.

\section{Discussion}

\section{GABAergic LC afferents in the peri-LC}

The present results show that GABAergic neurons in the peri-LC area synaptically innervate noradrenergic LC neurons. These inputs occur both within the LC nucleus, as revealed by WGA-Au retrograde labeling, and on LC dendrites in the peri-LC region, as revealed by ultrastructural analysis and PRV labeling. These GABA neurons receive a number of symmetric as well as asymmetric synaptic afferents, indicating that they serve to integrate different inputs. Previous studies have shown that this peri-LC region receives inputs from a large number of areas that do not project to, or only weakly innervate, the LC nucleus proper (AstonJones, in press; Aston-Jones et al., 1991, 1995). As GABA strongly inhibits impulse activity of LC neurons (Ennis and Aston-Jones, 1989; Shefner and Osmanovic, 1991), these results indicate that peri-LC GABAergic neurons may provide an inhibitory interneuron population for the LC noradrenergic system.

Previous studies have reported GABA neurons within the LC nucleus itself, but counts revealed this to be a small minority of cells compared with the noradrenergic population, numbering only $\sim 8 \%$ of neurons (Ijima and Ohtomo, 1988). Other studies have reported GABA neurons in the peri-LC region, and speculated that these cells may provide input to LC neurons (Jones, 1991; Van Bockstaele, 1998; Jasmin et al., 2003). Our results agree with that hypothesis. These peri-LC GABA neurons are much more numerous than those within the LC and, considered with the extensive afferent input to the pericerulear area, it appears that these cells represent an interneuron pool with substantial regulatory influences over LC activity.

Our results reveal that a large percentage of neurons retro-
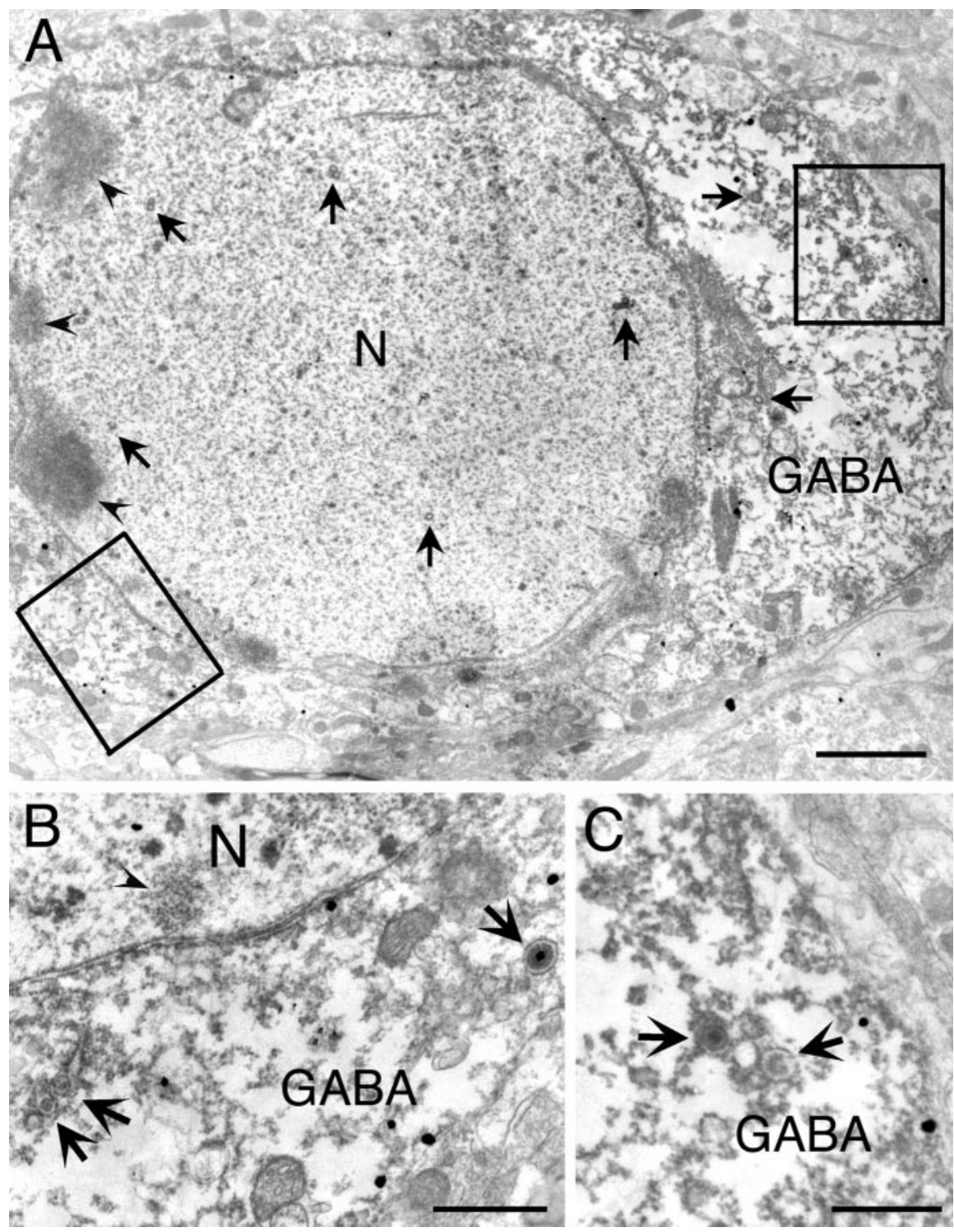

Figure 7. Ultrastructure of GABAergic LC afferents. EM photomicrograph of a neuron in the rostroventral peri- $L C$ immunolabeled for PRV (stained with DAB) and GABA (stained with silver-intensified gold). High-power images in $B$ and ( are taken from $B$, indicative of PRV infection (at arrowheads). Note also the cytoplasmic virions (indicated by arrows in $B$ and $C$ ), and abundant GABA staining in the cytoplasm. Scale bars: $A, 2 \mu \mathrm{m} ; B, C, 0.5 \mu \mathrm{m}$.

gradely labeled in the peri-LC from injections of tracers in the LC nuclear core stain for markers of GABA. Our light microscopic analysis revealed that $\sim 45 \%$ of retrogradely labeled neurons were $\mathrm{GABA}+$, whereas ultrastructural analyses indicated that $\sim 77 \%$ of LC afferents were GABAergic. This difference may reflect a higher sensitivity for immunohistochemical labeling in ultrastructural analyses compared with light microscopic observations. It is also possible that this result reflects sampling differences using these techniques, consistent with our anecdotal observation that double-labeled neurons in our light microscopic analyses often occurred in clusters.

\section{PRV as a tool for identifying inputs to distal LC dendrites}

PRV is a critical tool for investigating inputs to distal LC dendrites in the peri-LC region. Previous work has shown that the Bartha strain of PRV moves selectively retrogradely (Jasmin et al., 1997; O’Donnell, 1997; Card et al., 1998; Pickard et al., 2002), and 


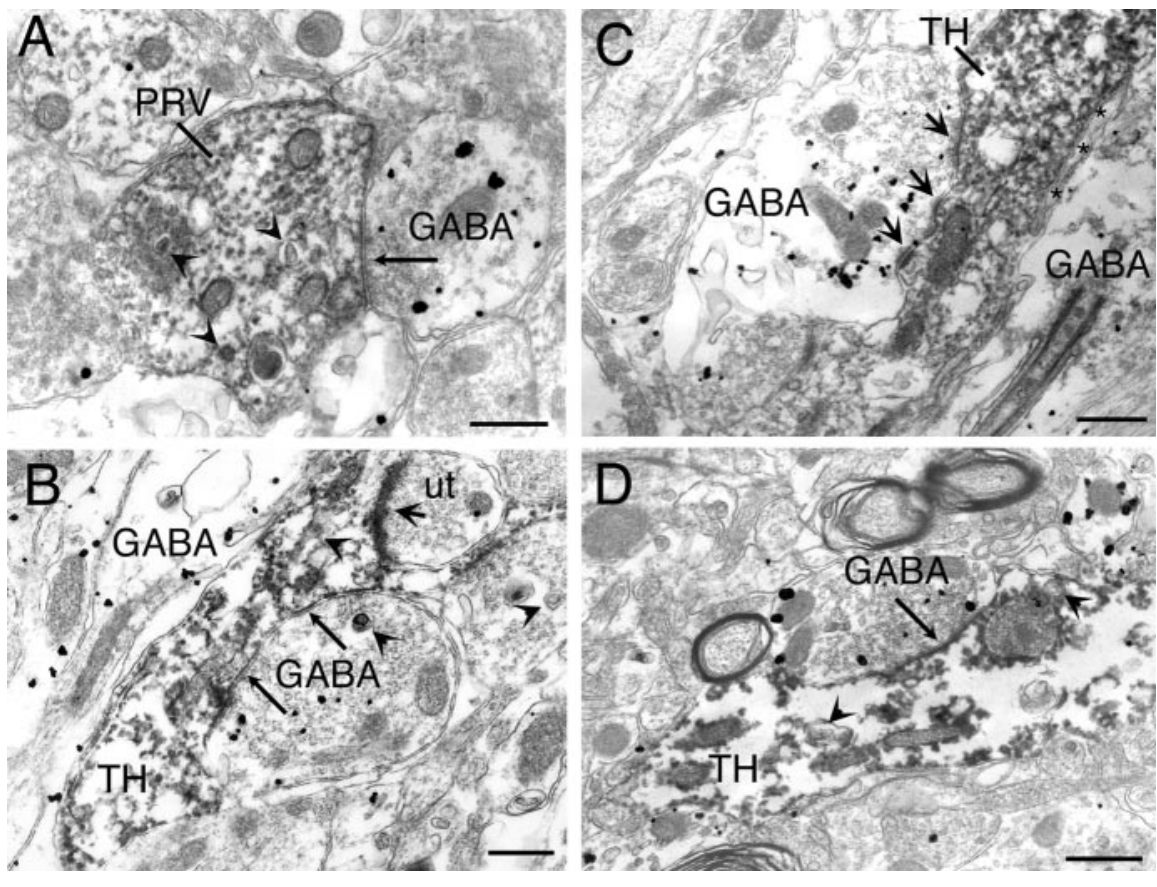

Figure 8. GABAergic terminals contacting PRV- or TH-labeled dendrites. A, High-power EM photomicrograph of an area in the rostroventral peri-LC immunolabeled for PRV (stained with DAB) and GABA (stained with silver-intensified gold). The dendrite in the center contains PRV virions (arrowheads) and receives a symmetric synapse (long arrow) from a GABA + terminal-containing pleomorphic vesicles. This is consistent with inhibitory influences of GABA neurons onto $\mathrm{LC}$ dendrites in the peri- $\mathrm{LC}$ region. $B$, High-power EM photomicrograph from an area in the rostroventral peri-LC immunolabeled with $\mathrm{TH}$ (stained with DAB) and GABA (stained with silver-intensified gold). The $\mathrm{TH}+$ dendrite receives symmetric synaptic contacts (long arrows) from a GABA + terminal. Note also that both the TH dendrite and the GABA terminal contain viral particles (arrowheads). This indicates PRV transfer from $\mathrm{TH}$ dendrites to $\mathrm{GABA}+$ afferent terminals in the peri- $\mathrm{LC}$ region. The $\mathrm{TH}$ dendrite also receives an asymmetric synaptic input (short arrow) from an unlabeled terminal (ut). C, Similar section as in $B$, showing multiple synaptic contacts of a GABA terminal with a $\mathrm{TH}+$ dendrite (arrows). Although the immunoperoxidase reaction in the postsynaptic dendrite makes classification of synapse symmetry difficult, it appears that two of the three contacts are symmetrical, whereas the postsynaptic density of the third contact is more prominent. Serial analysis would be required to make a definitive determination of the synapse morphology. Asterisks identify glial processes interposed between TH and GABA dendrites. D, High-power EM photomicrograph from an area in the rostroventral peri-LC immunolabeled with TH (stained with $D A B$ ) and GABA (stained with silver-intensified gold). A GABA terminal synapses onto a TH dendrite that contains PRV capsids (arrowheads). DAB reaction product underlies the symmetrical synaptic contact (long arrow). Scale bars, $0.5 \mu \mathrm{m}$.

viral transfer occurs only between neurons, not from glia to neurons, and occurs only at synapses (Card et al., 1993; Card and Enquist, 1995). Studies have also shown that PRV Bartha is transported distally along dendrites of neurons and can exit distal dendrites to infect afferent neurons (Card et al., 1993; Standish et al., 1995). The present study demonstrates that PRV injected into the LC nucleus is transported out to distal dendrites that reside in the peri-LC region, where the virus can trans-synaptically retrogradely invade and label afferents to those distal dendrites. Similar injections of conventional retrograde tracers into the LC would also label peri-LC neurons that project into the LC nucleus proper, but would leave unlabeled inputs to distal LC dendrites outside of the LC nucleus. Our ultrastructural analysis confirms that many GABA inputs to LC dendrites occur outside of the LC proper, in the peri-LC area.

Previous analysis of the intracellular transport of PRV in neurons of the dorsal motor vagal (DMV) nucleus infected by retrograde transport of virus from the stomach demonstrated that progeny virus trafficked into the distal dendrites of DMV neurons (Card et al., 1993). This has also been shown to be true of pyramidal cells of the prefrontal cortex retrogradely infected by injection of PRV into the nucleus accumbens (Carr et al., 1999). Data reported in the present study also demonstrated that virus and viral antigens were present in the most distal branches of LC neurons. Nevertheless, the temporal sequence of infection observed here at 23 and $35 \mathrm{hr}$ after injection suggests that there may be two projection-specific populations of GABA neurons in the peri-LC. (1) The equivalence of infected neurons observed at $23 \mathrm{hr}$ with the relative number and distribution of WGA-Au labeled neurons indicate that at least some of the peri-LC GABA neurons project directly into the nuclear core of LC. These neurons would be infected by direct uptake of PRV within the nuclear core. (2) The temporally delayed increase in peri-LC neurons seen at $35 \mathrm{hr}$ is consistent with the additional time that would be necessary for viral replication, intracellular trafficking, and transneuronal infection of neurons that synapse on the dendrites of infected LC neurons. It is also possible that the neurons in the peri-LC became infected via an indirect route involving another LC afferent as a relay. Note also that even distant direct inputs to the LC are strongly labeled by PRV at $24 \mathrm{hr}$ after injection (Aston-Jones and Card, 2000; Aston-Jones et al., 2001). However, the synaptology revealed in our ultrastructural analysis provides direct evidence of synaptic connections between peri-LC GABA neurons and LC dendrites. Collectively, these data provide strong evidence supporting the conclusion that a population of GABA neurons in the peri-LC exerts regulatory control over the LC via projections into the nucleus proper and through synaptic input to the extranuclear LC dendritic arbor.

Although many peri-LC neurons labeled by PRV or WGA-Au from LC injections are GABA+, many others do not stain for GABA. Previous studies have identified neurons containing other transmitters in this region, including serotonin, acetylcholine, and corticotropin-releasing factor (Aston-Jones et al., 1995). Additional studies are needed to determine the transmitter that these neurons may use to communicate with LC neurons.

\section{Inputs to GABAergic interneurons in the peri-LC: implications for regulation of LC function}

There are prominent inputs to the peri-LC region from areas that influence LC activity but do not have dense projections into the cell-dense region of the LC. For example, the medial prefrontal cortex (mPFC), dorsomedial hypothalamus, medial preoptic area, dorsal raphe, and central amygdala all project densely to the medial peri-LC region studied here but relatively little, if at all, to the LC nucleus proper (for review, see Aston-Jones, in press; Aston-Jones et al., 1991, 1995). The reason for this segregated input pattern was not clear in previous work. It is possible that at least some of these peri-LC afferents are contacting dendrites of LC neurons that extend outside of the nucleus. However, this would not explain why these inputs would not also project into the LC nucleus proper, where both distal and proximal dendrites 
exist. The present results provide an alternative explanation, such that inputs that integrate in an inhibitory interneuron pool before influencing the LC would preferentially project to the peri-LC area identified here as a potential major GABAergic interneuron population. These results call for additional physiological studies to test this hypothesized role of peri-LC GABA neurons.

The existence of an interneuron pool within the dendritic fields of the $\mathrm{LC}$ raises a number of issues regarding the way in which the functional output of this important brainstem nucleus is regulated. As noted previously, the extensive pericerulear dendritic field surrounding the nucleus proper has prompted a reevaluation of the synaptic influences on LC neurons. Chief among these considerations is the observation of afferents to the peri-LC that only minimally impinge on the cell-dense region of the LC. It is also noteworthy that LC dendrites accumulate in two distinct regions that appear to receive different populations of afferents. This observation suggests a functional topography, in which different populations of LC neurons may be subject to different regulatory influences. The possibility that a population of inhibitory interneurons serves to integrate and process some of these inputs suggests another level of dynamic regulatory capacity that would have important implications for understanding the way in which the activity of the LC is modulated. In this regard, it would be important to gain additional insight into the distribution of afferents on LC neurons and this putative interneuron pool.

\section{Summary}

In sum, the present results reveal a possible pool of local GABAergic inhibitory interneurons for the LC. Previous studies found that direct inputs to the LC nucleus are restricted to a small number of major afferents, and that additional inputs innervate distal LC dendrites outside of the LC nucleus proper. The present results expand the afferent circuit understanding of the LC system by indicating that the nu-

merous inputs to the peri-LC region may also innervate local neurons that in turn innervate LC cells. These findings call for additional studies to better understand how information is integrated in these local neurons to influence LC function.

\section{References}

Aston-Jones G (2004) Locus coeruleus, A5 and A7 noradrenergic cell groups. In: The rat nervous system, Ed 3 (Paxinos G, ed). Orlando: Academic, in press.

Aston-Jones G, Card JP (2000) Use of pseudorabies virus to delineate multisynaptic circuits in brain: opportunities and limitations. J Neurosci Methods 103:51-61.
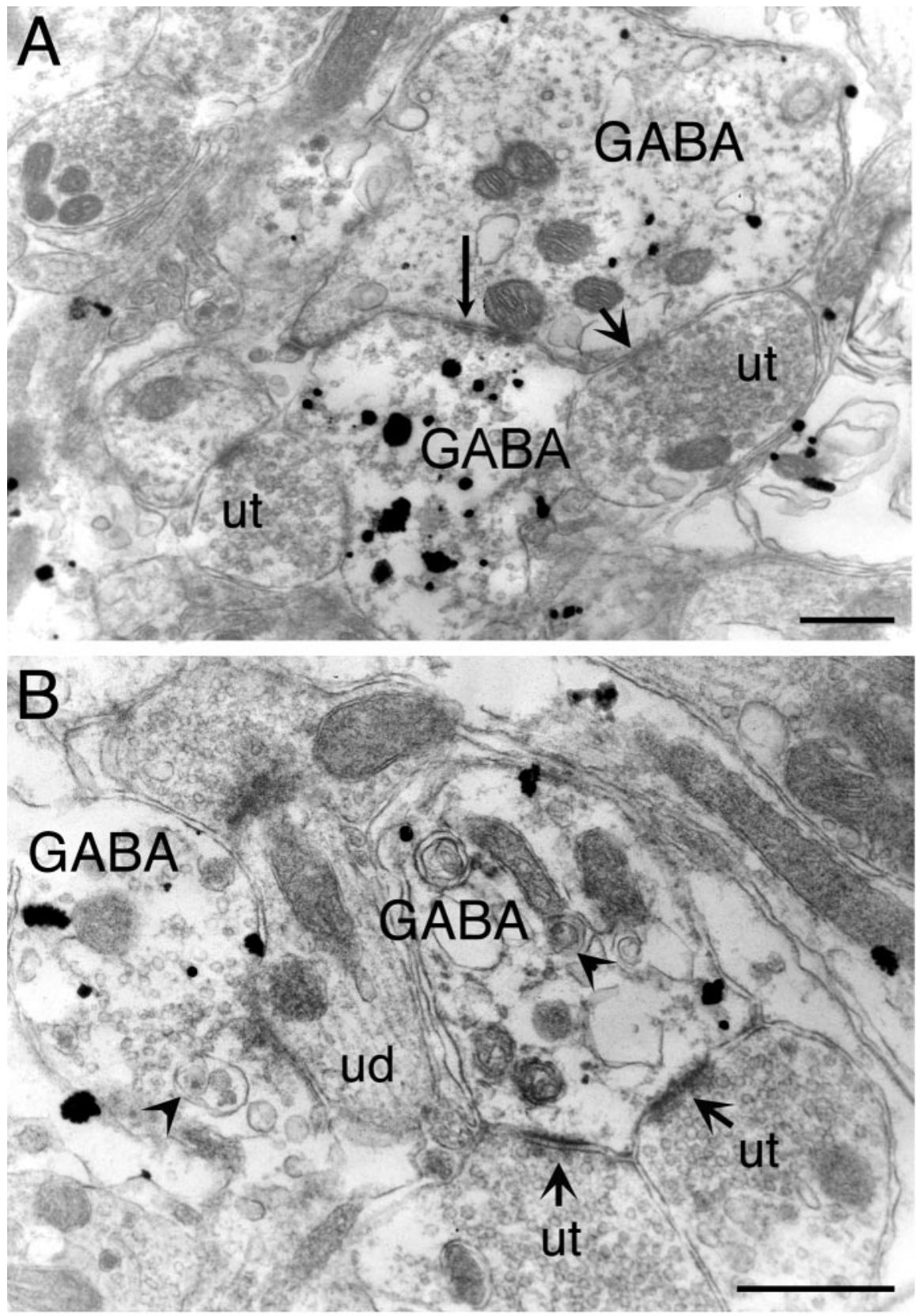

Figure 9. Peri-LC GABA neurons receive numerous synaptic inputs. High-power EM photomicrographs from the rostroventral peri-LC dendritic zone immunolabeled for GABA (stained with silver-intensified gold). A, A GABAergic dendrite receives synaptic input from GABA and non-GABA terminals. The contact from the GABA terminal is symmetrical in character (long arrow), whereas the unlabeled terminal (ut) forms an asymmetrical contact (short arrow). B, A GABAergic dendrite receives asymmetric synapses (short arrows) from unlabeled non-GABA terminals (ut). A labeled GABA terminal also forms a symmetrical contact with an unlabeled dendrite (ud). Both the GABAergic dendrite and GABAergic terminal contain PRV virions (arrowheads). Scale bars, $0.5 \mu \mathrm{m}$.

Aston-Jones G, Foote SL, Bloom FE (1984) Anatomy and physiology of locus coeruleus neurons: functional implications. In: Norepinephrine: frontiers of clinical neuroscience, Vol 2 (Ziegler M, Lake CR, eds), pp 92-116. Baltimore: Williams and Wilkins.

Aston-Jones G, Ennis M, Pieribone VA, Nickell WT, Shipley MT (1986) The brain nucleus locus coeruleus: restricted afferent control of a broad efferent network. Science 234:734-737.

Aston-Jones G, Shipley MT, Chouvet G, Ennis M, Van Bockstaele EJ, Pieribone V, Shiekhattar R, Akaoka H, Drolet G, Astier B, Charlety P, Valentino R, Williams JT (1991) Afferent regulation of locus coeruleus neurons: anatomy, physiology and pharmacology. Prog Brain Res 88:47-75. Aston-Jones G, Shipley MT, Grzanna R (1995) The locus coeruleus, A5 and 
A7 noradrenergic cell groups. In: The rat nervous system, Ed 2 (Paxinos G, ed), pp 183-214. New York: Academic.

Aston-Jones G, Rajkowski J, Cohen J (2000) Locus coeruleus and regulation of behavioral flexibility and attention. Prog Brain Res 126:165-182.

Aston-Jones G, Chen S, Zhu Y, Oshinsky M (2001) A neural circuit for circadian regulation of arousal. Nat Neurosci 4:732-738.

Berod A, Chat M, Paut L, Tappaz M (1984) Catecholaminergic and GABAergic anatomical relationship in the rat substantia nigra, locus coeruleus, and hypothalamic median eminence: immunocytochemical visualization of biosynthetic enzymes on serial semithin plastic-embedded sections. J Histochem Cytochem 32:1331-1338.

Berridge CW, Waterhouse BD (2003) The locus coeruleus-noradrenergic system: modulation of behavioral state and state-dependent cognitive processes. Brain Res Brain Res Rev 42:33-84.

Card JP, Enquist LW (1995) Neurovirulence of pseudorabies virus. Crit Rev Neurobiol 9:137-162.

Card JP, Rinaman L, Schwaber JS, Miselis RR, Whealy ME, Robbins AK, Enquist LW (1990) Neurotropic properties of pseudorabies virus: uptake and transneuronal passage in the rat CNS. J Neurosci 10:1974-1994.

Card JP, Rinaman L, Lynn RB, Lee B-H, Meade RP, Miselis RR, Enquist LW (1993) Pseudorabies virus infection of the rat CNS: ultrastructural characterization of viral replication, transport, and pathogenesis. J Neurosci 13:2515-2539.

Card JP, Levitt P, Enquist LW (1998) Different patterns of neuronal infection after intracerebral injection of two strains of pseudorabies virus. J Virol 72:4434-4441.

Card JP, Enquist LW, Moore RY (1999) Neuroinvasiveness of pseudorabies virus injected intracerebrally is dependent on viral concentration and terminal field density. J Comp Neurol 407:438-452.

Carr DB, O'Donnell P, Card JP, Sesack SR (1999) Dopamine terminals in the rat prefrontal cortex synapse on pyramidal cells that project to the nucleus accumbens. J Neurosci 19:11049-11060.

Cedarbaum JM, Aghajanian GK (1978) Afferent projections to the rat locus coeruleus as determined by a retrograde tracing technique. J Comp Neurol 178:1-16.

Chan J, Aoki C, Pickel VM (1990) Optimization of differential immunogold-silver and peroxidase labeling with maintenance of ultrastructure in brain sections before plastic embedding. J Neurosci Methods 33:113-127.

Chen S, Ming X, Miselis R, Aston-Jones G (1999) Characterization of transsynaptic tracing with central application of pseudorabies virus. Brain Res 838:171-183.

Drolet G, Van Bockstaele EJ, Aston-Jones G (1992) Robust enkephalin innervation of the locus ceruleus from the rostral medulla. J Neurosci 12:3162-3174.

Ennis M, Aston-Jones G (1988) Activation of locus ceruleus from nucleus paragigantocellularis: a new excitatory amino acid pathway in brain. J Neurosci 8:3644-3657.

Ennis M, Aston-Jones G (1989) GABA-mediated inhibition of locus ceruleus from the dorsomedial rostral medulla. J Neurosci 9:2973-2981.

Enquist LW, Husak PJ, Banfield BW, Smith GA (1999) Infection and spread of alphaherpesviruses in the nervous system. Adv Viral Res 51:237-347.

Horvath TL, Peyron C, Sabrina D, Ivanov A, Aston-Jones G, Kilduff T, van den Pol AN (1999) Strong hypocretin (orexin) innervation of the locus coeruleus activates noradrenergic cells. J Comp Neurol 415:145-159.

Ijima K, Ohtomo K (1988) Immunocytochemical study using a GABA antiserum for the demonstration of inhibitory neurons in the rat locus coeruleus. Am J Anat 181:43-52.

Jasmin L, Burkey AR, Card JP, Basbaum AI (1997) Transneuronal labeling of a nociceptive pathway, the spino- (trigeminal-) parabrachioamygdaloid, in the rat. J Neurosci 17:3751-3765.

Jasmin L, Rabkin SD, Granato A, Boudah A, Ohara PT (2003) Analgesia and hyperalgesia from GABA-mediated modulation of the cerebral cortex. Nature 424:316-320.

Jones BE (1991) Noradrenergic locus coeruleus neurons: their distant connections and their relationship to neighboring neurons of the central gray and reticular formation. Prog Brain Res 88:15-30.

Mugnaini E, Oertel WH (1985) An atlas of the distribution of GABAergic neurons and terminals in the rat CNS as revealed by GAD immunohistochemistry. In: Handbook of chemical neuroanatomy (Bjorklund A, Hokfelt T, eds), pp 436-608. Amsterdam: Elsevier.

O’Donnell P (1997) Interconnected parallel circuits between rat nucleus accumbens and thalamus revealed by retrograde transsynaptic transport of pseudorabies virus. J Neurosci 17:2143-2167.

Oertel WH, Tappaz ML, Berod A, Mugnaini E (1982) Two-color immunohistochemistry for dopamine and GABA neurons in rat substantia nigra and zona incerta. Brain Res Bull 9:463-474.

Pickard GE, Smeraski CA, Tomlinson CC, Banfield BW, Kaufman J, Wilcox CL, Enquist LW, Sollars PJ (2002) Intravitreal injection of the attenuated pseudorabies virus PRV Bartha results in infection of the hamster suprachiasmatic nucleus only by retrograde transsynaptic transport via autonomic circuits. J Neurosci 22:2701-2710.

Pickel VM, Joh TH, Field PM, Becker CG, Reis DJ (1975) Cellular localization of tyrosine hydroxylase by immunohistochemistry. J Histochem Cytochem 23:1-12.

Pieribone VA, Aston-Jones G, Bohn MC (1988) Adrenergic and nonadrenergic neurons of the $\mathrm{C} 1$ and $\mathrm{C} 3$ areas project to locus coeruleus: a fluorescent double labeling study. Neurosci Lett 85:297-303.

Pollard H, Llorens-Cortes C, Barbin G, Garbarg M, Schwartz JC (1978) Histamine and histidine decarboxylase in brain stem nuclei: distribution and decrease after lesions. Brain Res 157:178-181.

Rinaman L, Card JP, Schwaber JS, Miselis RR (1989) Ultrastructural demonstration of a gastric monosynaptic vagal circuit in the nucleus of the solitary tract in rat. J Neurosci 9:1985-1996.

Shefner SA, Osmanovic SS (1991) GABAA and GABAB receptors and the ionic mechanisms mediating their effects on locus coeruleus neurons. Prog Brain Res 88:187-195.

Shipley MT, Fu L, Ennis M, Liu W, Aston-Jones G (1996) Dendrites of locus coeruleus neurons extend preferentially into two pericoerulear zones. J Comp Neurol 365:56-68.

Standish A, Enquist LW, Miselis RR, Schwaber JS (1995) Dendritic morphology of cardiac related medullary neurons defined by circuit-specific infection by a recombinant pseudorabies virus expressing $\beta$-galactosidase. J Neurovirol 1:359-368.

Steininger TL, Gong H, McGinty D, Szymusiak R (2001) Subregional organization of preoptic area/anterior hypothalamic projections to arousalrelated monoaminergic cell groups. J Comp Neurol 429:638-653.

Swanson LW (1976) The locus coeruleus: a cytoarchitectonic, Golgi and immunohistochemical study in the albino rat. Brain Res 110:39-56.

Van Bockstaele EJ (1998) Morphological substrates underlying opioid, epinephrine and gamma-aminobutyric acid inhibitory actions in the rat locus coeruleus. Brain Res Bull 47:1-15.

Van Bockstaele EJ, Colago EEO, Aicher S (1998) Light and electron microscopic evidence for topographic and monosynaptic projections from neurons in the ventral medulla to noradrenergic dendrites in the rat locus coeruleus. Brain Res 784:123-138.

Whealy ME, Card JP, Meade RP, Robbins AK, Enquist LW (1991) Effect of brefeldin A on alphaherpesvirus membrane protein glycosylation and virus egress. J Virol 65:1066-1081. 